\title{
CREATING SUSTAINABLE URBAN LANDSCAPES: MAPPING WITH PLACEMAKER
}

\author{
M. SEPE \\ Consiglio Nazionale delle Ricerche, Rome, Italy. \\ Dipartimento di Progettazione Urbana e di Urbanistica, Università di Napoli Federico II, Naples, Italy.
}

\begin{abstract}
The new urban features are not easily identifiable and cannot be easily represented through traditional cartography and tools of representation. In order to explain such new sites and give new terms, several researchers have tested new methodologies, maps, multimedia images, hypertext and software that can render this complexity and permit readability. Starting from those premises, the aim of this study, carried out in the framework of a convention between Consiglio Nazionale delle Ricerche and Dipartimento di Progettazione Urbana e di Urbanistica, Università di Napoli Federico II, is to illustrate new methodological approaches for analysing and representing contemporary urban landscapes. In particular, in the context of the complex-sensitive approach and PlaceMaker method of analysis, a new software tool, currently under development, is presented. The PlaceMaker method identifies the elements of the urban landscape which contribute to the identification of places and are able to influence the cultural and sustainable city construction; such elements and the complexity of the places are represented in a complex map. Experimentation of the method has shown the necessity of the proposed PlaceMaker software tool, which is able to support the collection and management of a multimedia database, the implementation of the phases of PlaceMaker method, the construction of the interactive complex map, and the calculation of indices useful for the project of sustainable urban landscapes.
\end{abstract}

Keywords: complex mapping, contemporary urban transformations, multimedia software, PlaceMaker, sustainable urban landscapes.

\section{INTRODUCTION}

The new structure of the contemporary city is unpredictable and complex, especially given the continuous mix of cultures which brings new elements to the already multiple identity of places, breaking, mixing and recomposing the complexity of urban life (see Figs 1-6) [1-5]. In order to give suitable terms and explain such new sites, several academics are testing maps, words and multimedia images, hypertext and software that can render this complexity and permit readability, leading in most cases to new interpretations of urban features [6-8]. The study of aspects that do not univocally translate into objective facts must meet three criteria: scientificity, giving objective results and ensuring repeatability of the method in different contexts; updating capability, with the possibility to add new data and modify existing data; and timing, so as to be able to use the results respecting the evolution of a sustainable programming and town planning process. Two main distinctions can be operated about the contemporary methods of analysis of the city places, concerning the theoretical approach and the type of instrument used to make explicit the results [9]. The principal types of approach to the analysis of the places are represented by the virtual [10], lateral [11], nomadic [12], multiscale [13] and configurational [14] approaches, that can be split into two categories. The first category, which includes the virtual, the lateral and the nomad, makes use of atlases, maps, schemes and videos to represent the products of their analyses; the second category, which includes the multiscale and the configurational approaches, is based on data processing tools and, in particular, software tools for the collection and management of the data.

Starting from those premises, the aim of this study, carried out in the framework of a convention between Consiglio Nazionale delle Ricerche and Dipartimento di Progettazione Urbana e di Urbanistica, Università di Napoli Federico II, is to illustrate the contemporary methodological approaches 


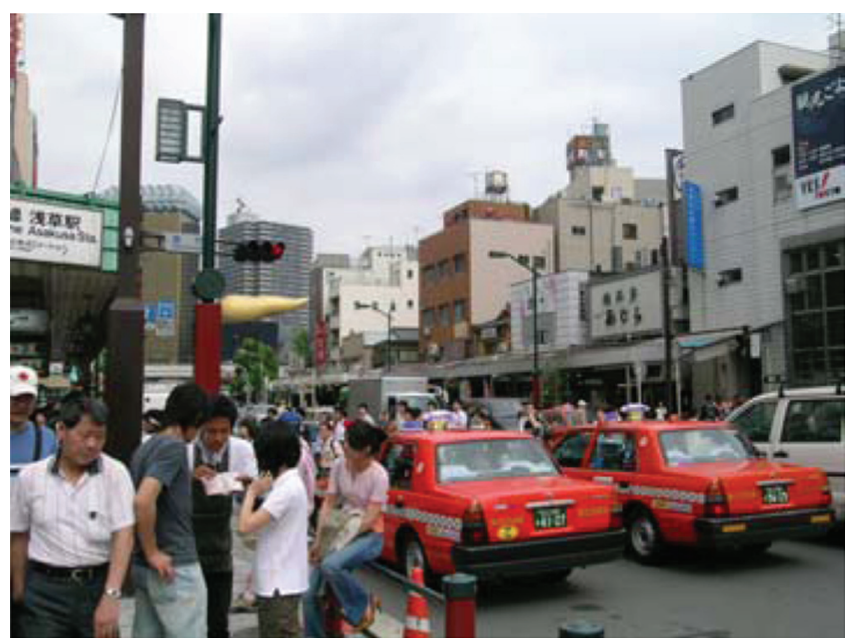

Figure 1: Tokyo, Asakusa area.

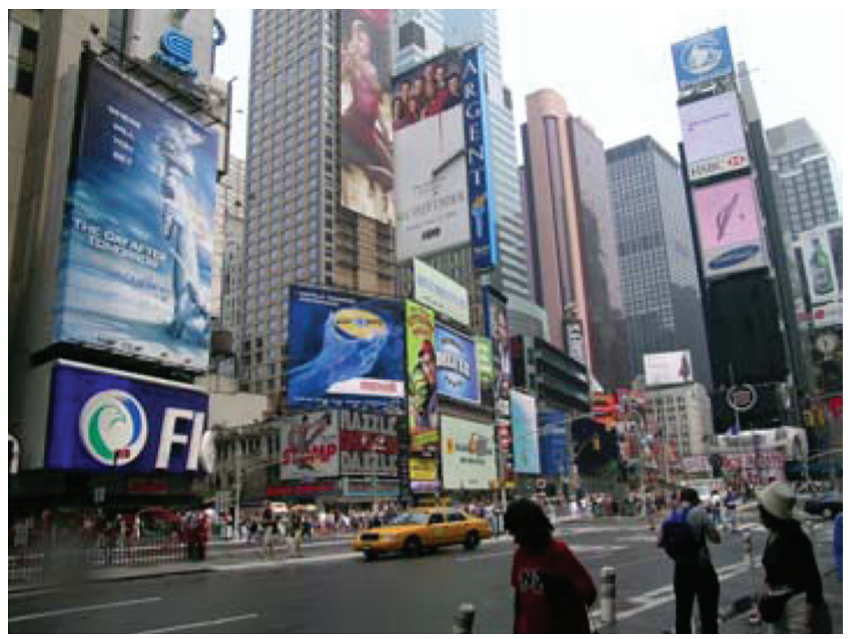

Figure 2: New York, Times Square.

to the urban analysis and tools for representation of the results. The study of these approaches has motivated the development of a new approach that can be defined as complex-sensitive based on the PlaceMaker method. The complex-sensitive approach can be included in the first category, with respect to the adopted methodological tools, and in the second category, as regards the supporting tool used for the analysis. The PlaceMaker method, conceived since 2001 and continuously elaborated and enriched during the experimentation started in 2002, has investigated urban areas in Europe and beyond, which are highly representative of the cities in which they are located, by testing the various tools used in the different phases of the analysis, the efficiency of the timing and the products of the various phases, in the process identifying the need for some integrations. This experimentation has 


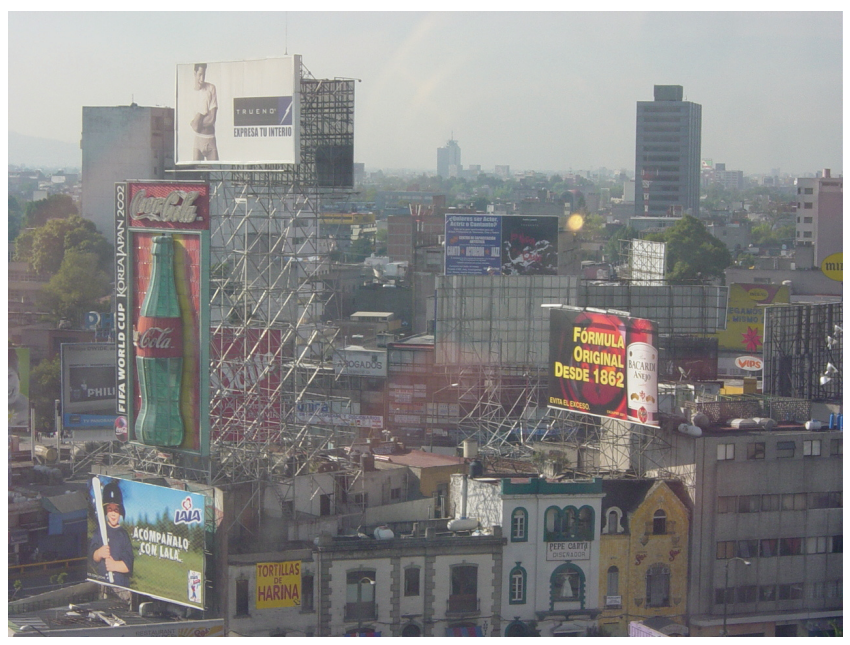

Figure 3: Mexico City, Zona Rosa.

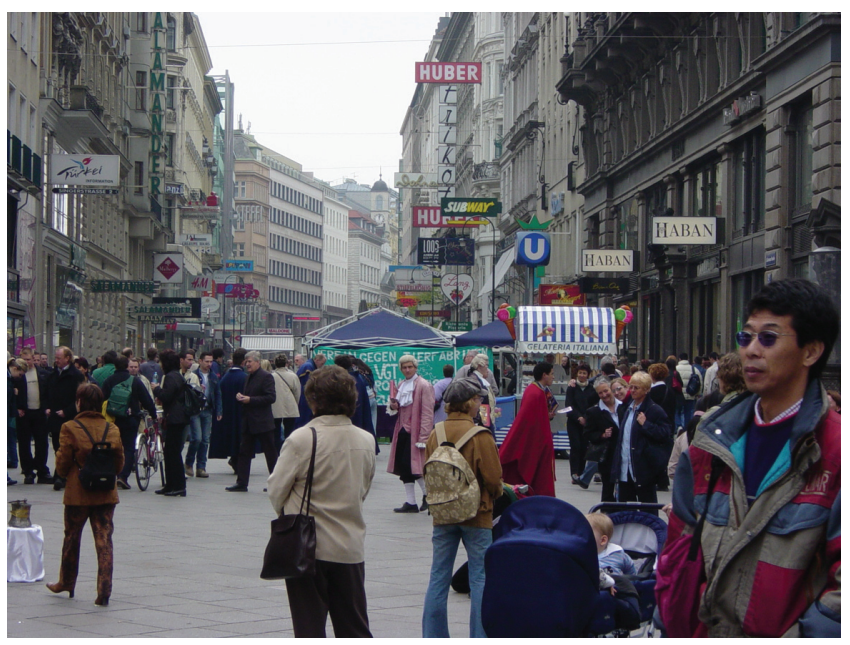

Figure 4: Vienna, Kartner Strasse.

shown the necessity of the PlaceMaker software tool, proposed in this article, which is able to support the implementation of the phases of the PlaceMaker method, the construction of the interactive complex map, and the calculation of indices useful for the analysis and the project of the sustainable urban landscape.

The article is organized as follows. Section 2 describes the categories of approaches with the most representative methods of analysis and the relative tools of representation. Section 3 explains the characteristics and functions of the PlaceMaker software. Finally, the conclusions are presented in Section 4. 


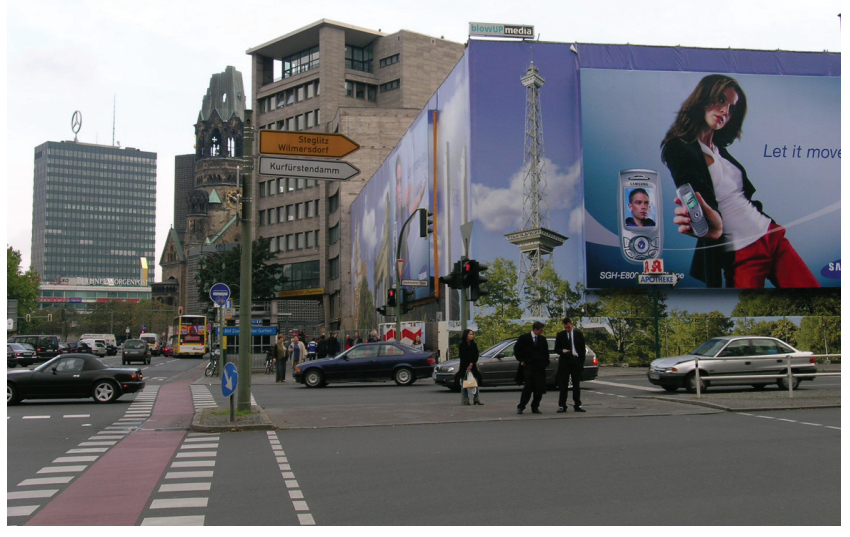

Figure 5: Berlin, Ku'damm.

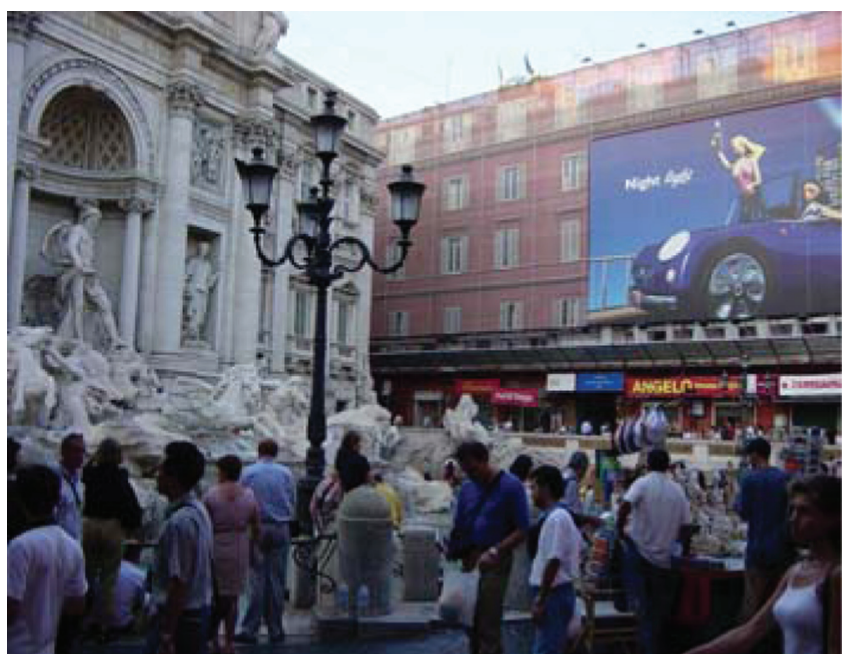

Figure 6: Rome, Fontana di Trevi.

\subsection{The virtual approach}

\section{CONTEMPORARY METHODS OF URBAN ANALYSIS}

The virtual approach to analysing urban places is an approach that finds its expression in the myriad sites created through the use of the network. These are spaces, squares, architecture, platforms and gateways which, despite borrowing terms from the constructed world, are not physical places, but are able to influence movement, behaviour and habits [10, 15-16] (see Figs 7-9). City of Bits by W.J. Mitchell is a study of a new type of city, an increasingly important system of virtual spaces interconnected by the information superhighway. 
The analysis proposed by Mitchell represents a sociological and cultural example of a reality dominated by the Internet, which we have experienced in the last few decades and which is now ready to change profoundly and indeed uncontrollably people's lives and the space in which they circulate. Architecture and urban planning are inserted in a context suggested by the digital telecommunications revolution so as to describe an invisible reality. The resulting map is a sort of virtual architecture whose concrete meaning lies in the virtual paths that we habitually take: a picture of the change in architectural and urban space and its users/inhabitants due to the technological innovations introduced by the Internet today and in the future on which to build the future of the city.

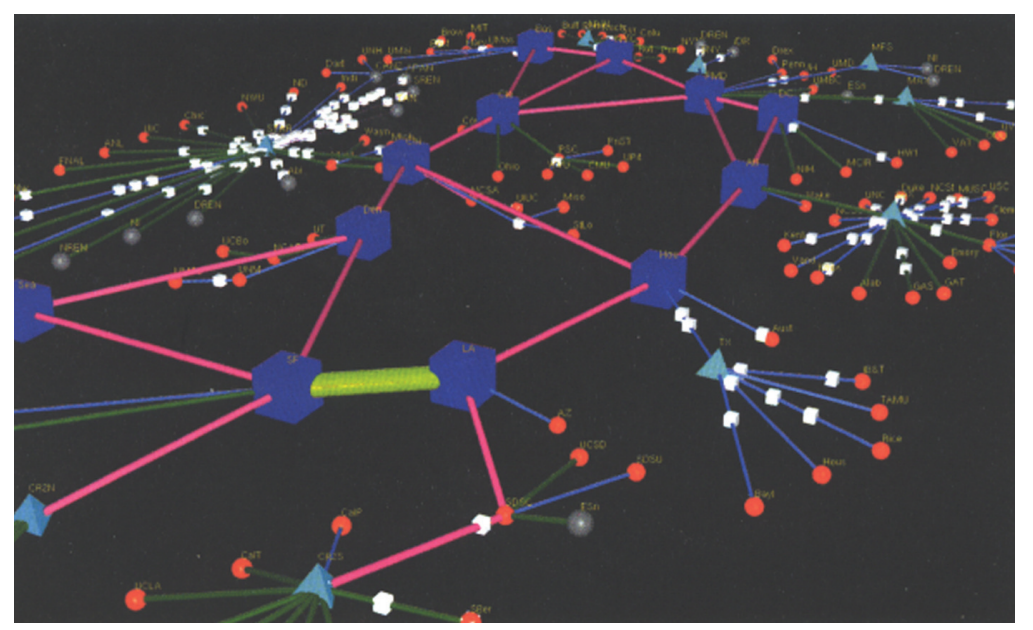

Figure 7: Jeff Brown, screen shot of a 3D model of the vBNS network connecting universities and laboratories in the USA [7].

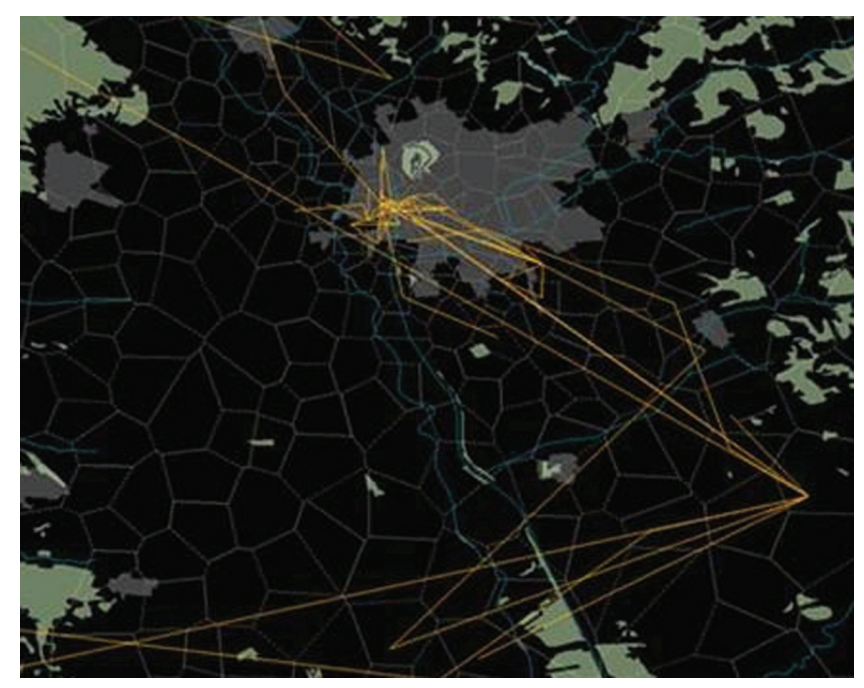

Figure 8: Carlo Ratti, mobile landscape map [15]. 


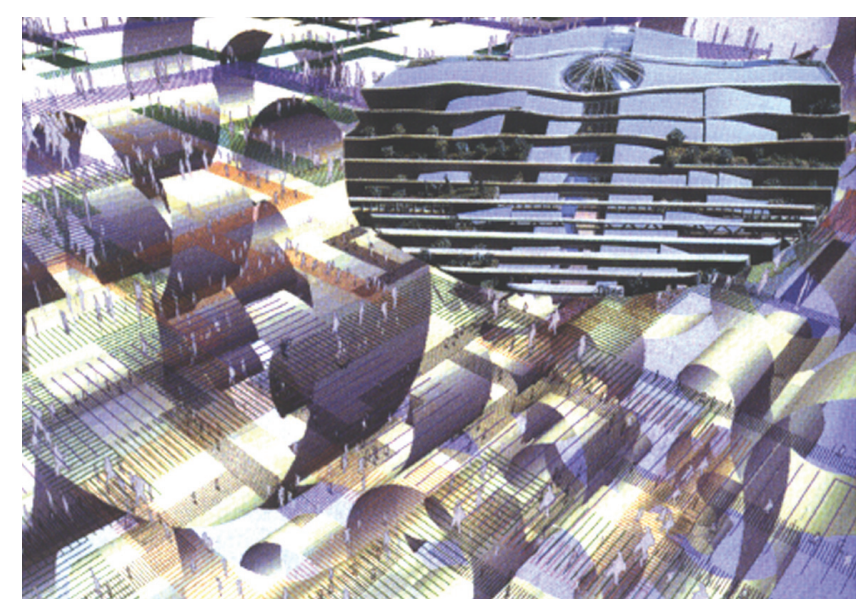

Figure 9: James Wines, model [16].

\subsection{The lateral approach}

The lateral approach is an approach to interpreting the urban landscape that presupposes a cross-sectional analytical approach to study an area from different points of view and on different scales of interpretation. Such an approach is also based on the perceptual, sociological or anthropological aspects, or on all three together. It is an analytical method whose foundations lie in the urban studies of Lynch and Cullen and results in maps, eclectic atlases, artworks and any tool deemed useful for representing the elements observed [17-21] (see Figs 10-12). Stefano Boeri with USE (Uncertain States of Europe) - a research program on contemporary Europe, studied the change in real time of contemporary space and investigated elements testifying to the changes in people's behaviour and the flow of goods and ideas in contemporary Europe [11]. The aim is to convert into comprehensible language the complexity of contemporary changes, offering new interpretational keys for surveying the urban landscape. The product is represented by eclectic atlases, which propose new ways to study the correspondence between space and society. Eclectic atlases produce temporary maps which represent places laterally, moving at the same time between physical and mental spaces.

\subsection{The nomadic approach}

The nomadic approach has its roots in the deambulations of Costant and the paths of the Situationists (see Figs 13 and 14) and is founded on the study of an area based on knowledge gained through direct experience. The survey method created by the Stalker group is to identify new operating categories for architecture through the action of walking; to wander in the city without control and unpredictably. The Stalker studies the area, paying attention to areas of rejection and abandonment, to voids and urban spaces undergoing change. Such surveys are developed on various levels and with different representations of the spaces that they call Actual Territories. These are marginal areas, places of memory, spaces for the comparison between nature and artifice, which are difficult to define, represent and project. They can only be known by direct experience, through the use of testimony rather than representation. The Stalker crosses areas on foot so as to have no support from mediating tools and in order to participate in their dynamics: it is a type of nomadic search for knowledge, without necessarily having to define the quest of knowledge, because the action of crossing is for the 


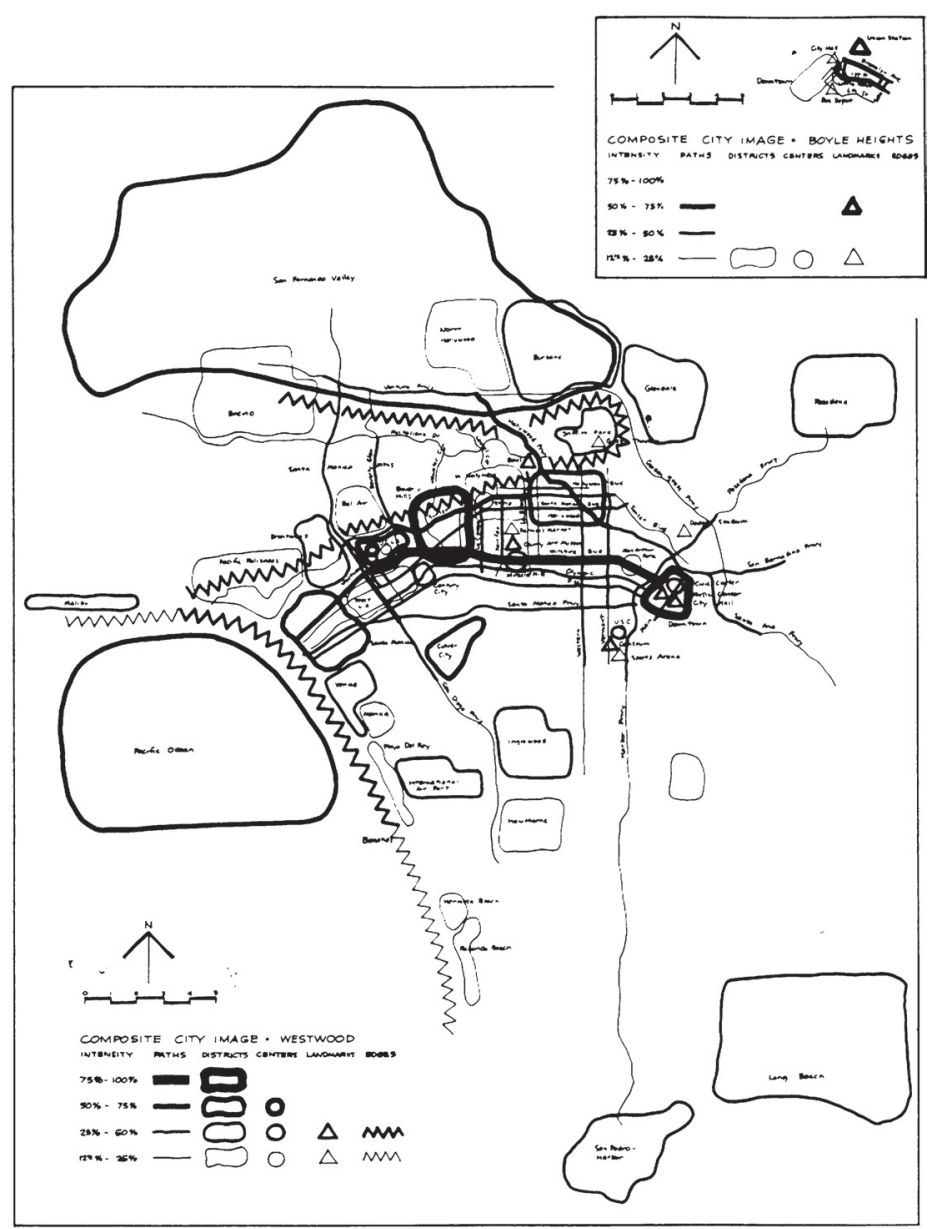

Figure 10: Kevin Lynch, view of Los Angeles [5].

Stalker already a creative action. The product is represented by a sort of map that is mobile like the area crossed and the tool used to cover it [22, 23].

\subsection{The multiscale approach}

The multiscale approach is an approach that presupposes knowledge and the attainment of a very large body of data from different sources able to interact and supply the answers required to interpret an area (see Figs 15 and 16). It is a type of approach that from some points of view may be described as the completion and extension of a GIS in a more dynamic and flexible form [13,24]. The analysis elaborated by the MVRDV group for the RhineRuhrCity research project is based on the idea that cities are complex organisms rich in inner connections. Moreover, cities must be able to ensure the coexistence of a set of diversified functions and facilities that entail enormous economic advantages. Smaller urban areas cannot offer the same complexity of facilities with respect to cities, which have the edge in terms of supply. In order to make the former competitive, the solution of the MVRDV lies 


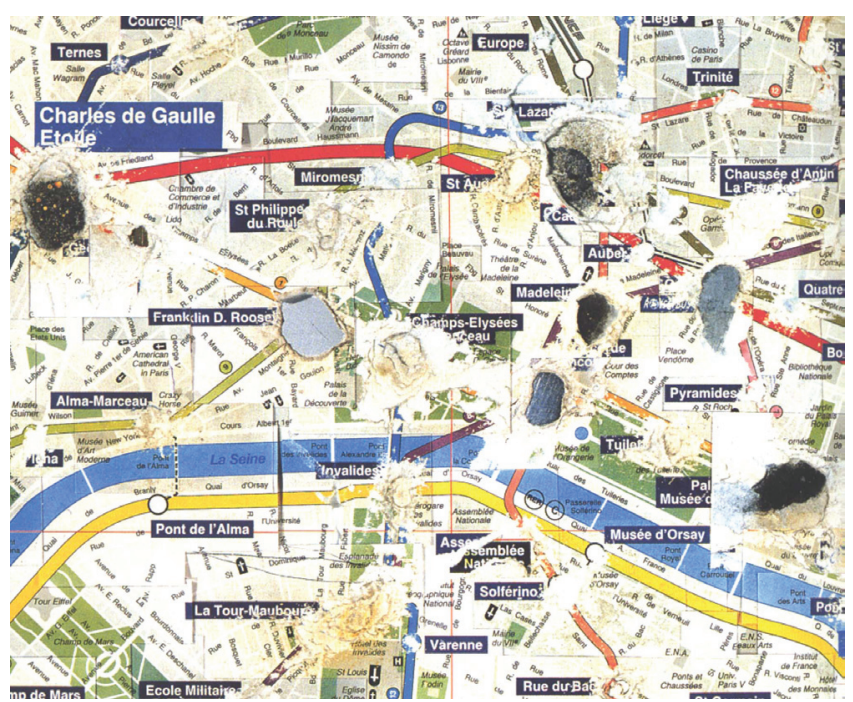

Figure 11: Paola Di Bello, la disparition, detail of the collage [8]

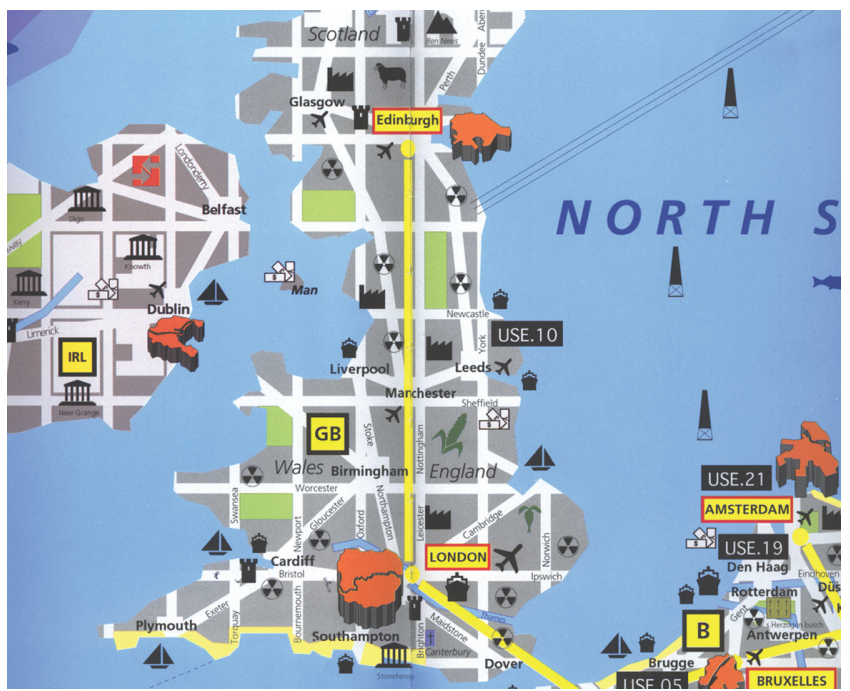

Figure 12: Stefano Boeri, USE, detail of eclectic atlases [11].

in networks, in the creation of complex systems of towns, cities and regions. Combining hard and soft resources intelligently, multicentre cities can be competitive because they combine the variety of resources of large centres with a better quality of life. The MVRDV has produced a set of software called 'The RegionMaker' to study the region of the Ruhr, that combines the functions of a search engine, a graphic interface, and a browser. These tools are able to collect demographic data and values supplied by the GIS and it is possible to consult maps, diagrams, access data banks, export satellite images, log on to the Internet, and use CAD planning. 


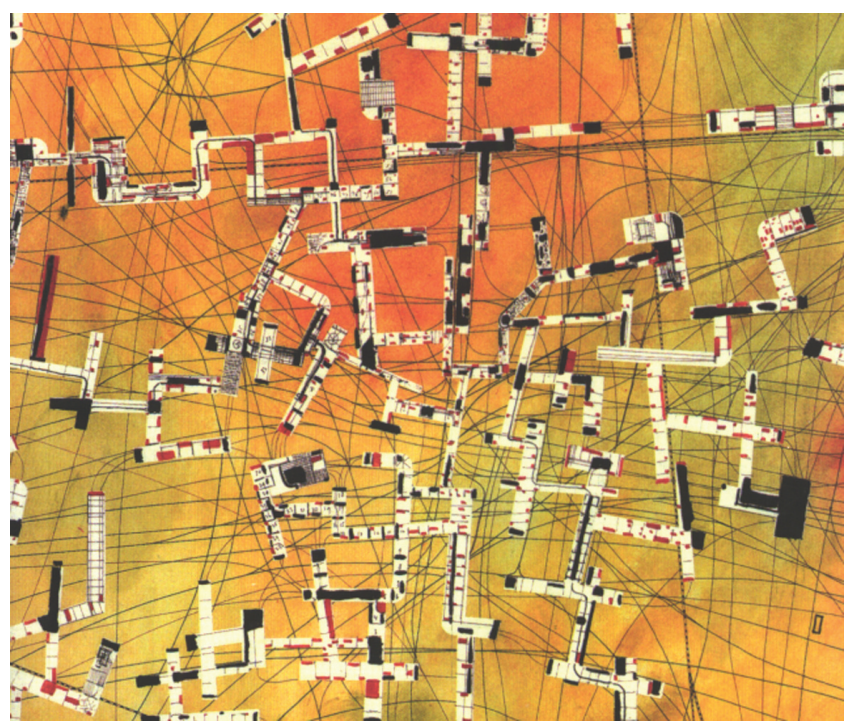

Figure 13: Costant, New Babylon [12].

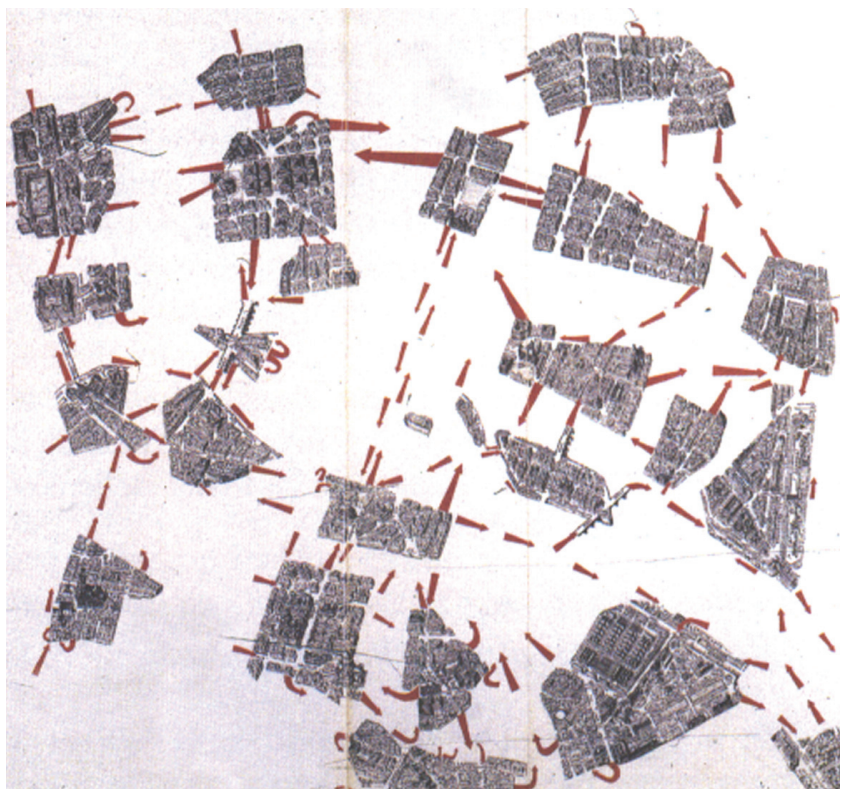

Figure 14: Guy Debord, Guide Psychogéographique de Paris [12].

\subsection{The configurational approach}

The configurational approach regards the study of a complex of inter-relations. As shown by Prof. Bill Hillier and his group work, the Space syntax research method controls the variables of physical complexity, treating built environments as systems of space and analysing them configurationally. 


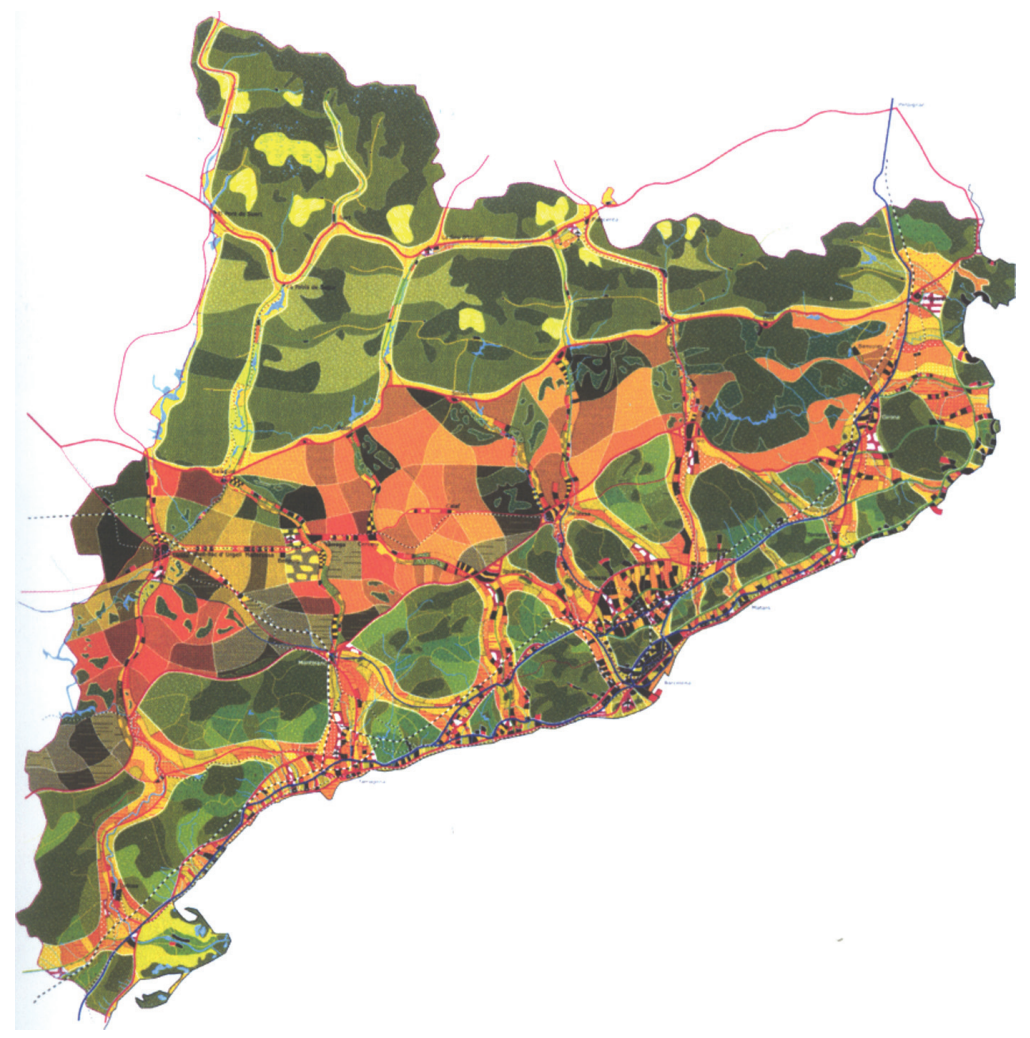

Figure 15: Manuel Gausa, HiperCatalunya [24].

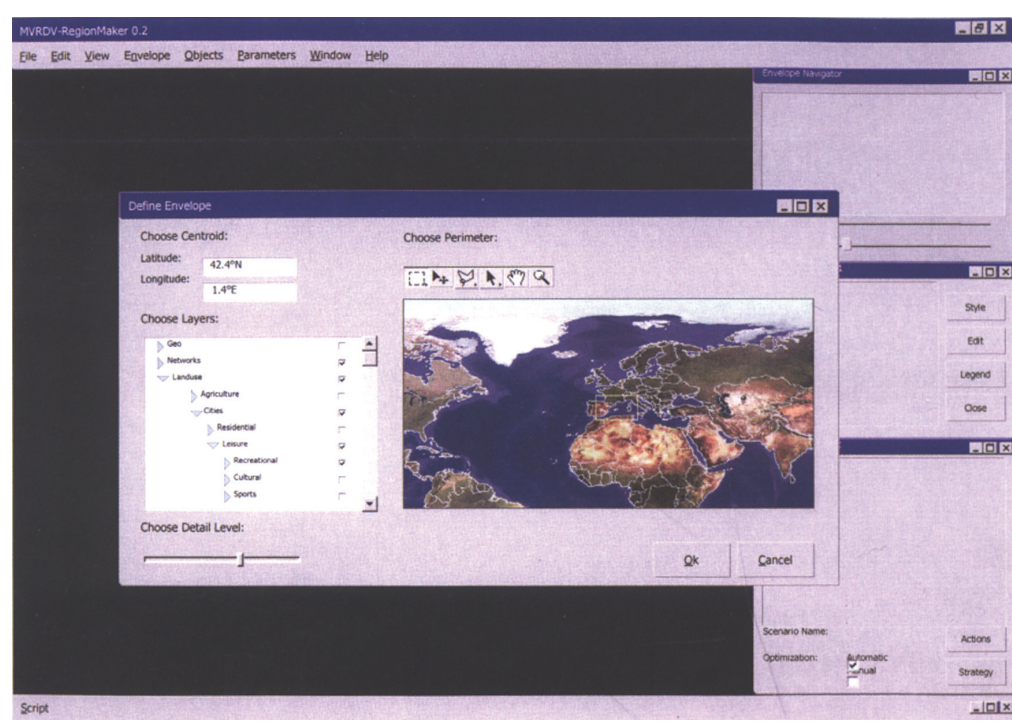

Figure 16: MVRDV, RegionMaker [24]. 
Furthermore, Hillier tries to uncover the patterns and structure of these variables. The most important aspect of Space syntax is represented by the set of methods for analysing patterns of space in the built environment to find spatial structures in cities and relate them to the way people move, stop, and interact $[14,25,26]$. Space syntax models analyse patterns of real space using simple mathematical tools that typically relate all elements to all others up to some limit, as far as this is possible. This method has been used by Hillier, including axial analysis, to observe the network of streets and walkways in cities, and visibility graph analysis to study patterns of visual fields in public spaces. The result is represented by an axial map of an urban area and its context (see Figs 17 and 18). Furthermore, a set of software tools has been created to perform Space syntax analysis. One of the most popular tools is Axman, an application to perform axial analysis on single buildings or entire cities.

\subsection{The complex-sensitive approach}

The complex-sensitive approach studies the urban place in all its complexity; it is sensitive, because it is open to all the stimuli provided by the places and seeks to identify and represent elements linked to features, which are both perceptive and objective, permanent and transitory [27-33]. The main method of analysis that represents this kind of approach is represented by PlaceMaker. The PlaceMaker method is a methodology for analysing the urban landscape designed to identify elements that do not feature in traditional mapping and which constitute the contemporary identity of the places, representing them in a complex map that provides a complex, but immediately understandable reading of the sites (see Fig. 19). PlaceMaker, unlike other approaches of analysis which study only one aspect of the site (perceptive, urban, etc.) or other multidisciplinary approaches which collect a lot of data with many difficulties to manage them, considers the places from all points of views, using different but comparable tools of relief, constituting an important instrument for sustainable urban construction.

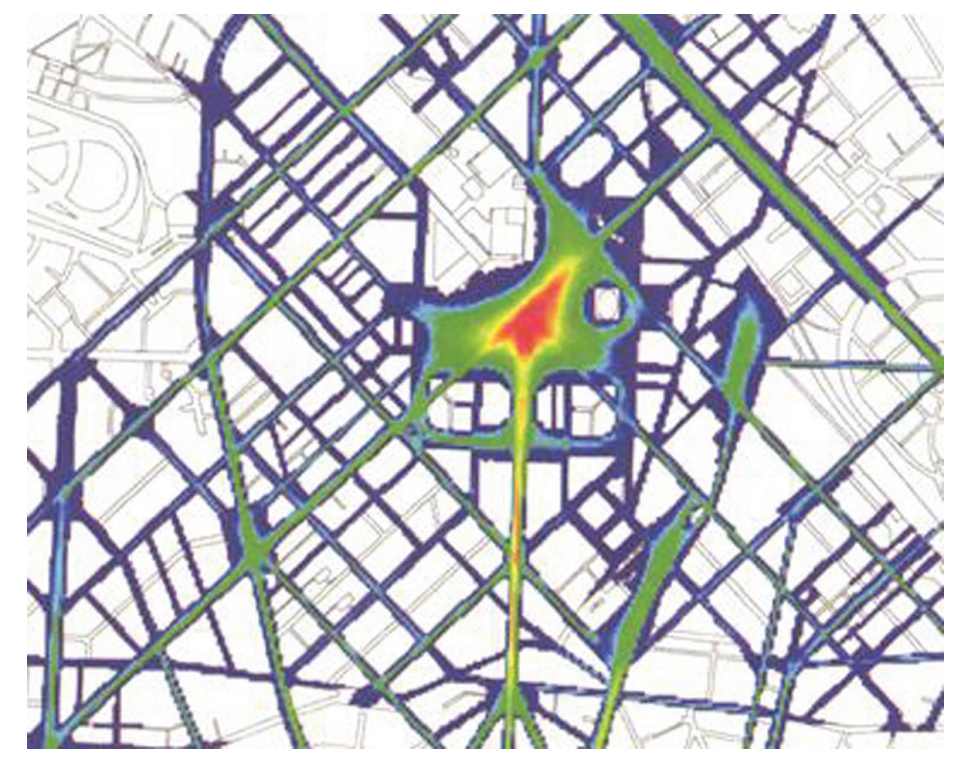

Figure 17: Bill Hillier, site in Milan showing spatial agent analysis [14]. 


\section{FACTORS INFLUENCING MOVEMENT}

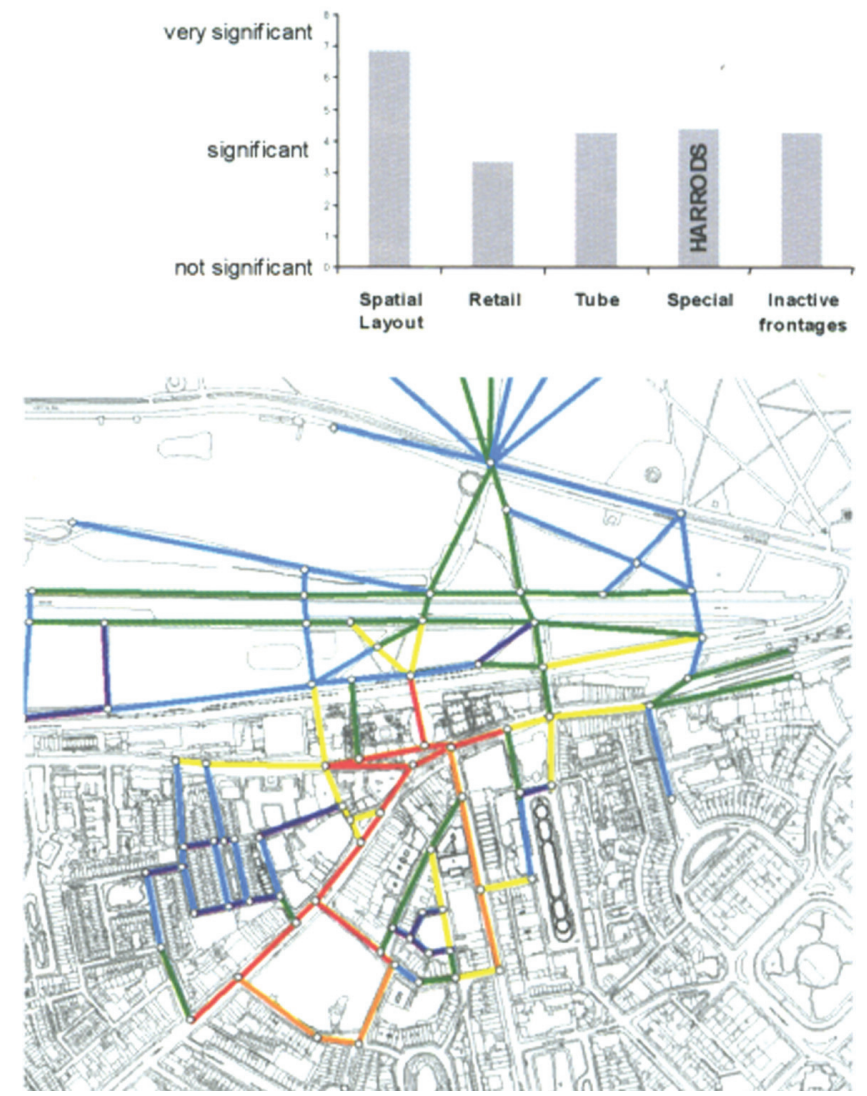

Figure 18: Bill Hillier, space syntax map [14].

This method assembles, elaborates and reconstructs the data deriving from surveys based on physical reconnaissance, sensory perceptions, graphical elaboration, photographic and video records, and sets this data against that provided by an overview of expectations, an analysis based on traditional cartography and a questionnaire given to local inhabitants.

In order to support the method of analysis in all its phases and the creation of the complex map, a specific software is under development. A useful reference in the creation of the software has been the search carried out on the products and their applications which connect the GIS with the multimedia potentiality, allow the construction of conceptual maps, or support the construction of urban interactive observatories and maps for the participation of citizens in the planning process [34-36].

\subsection{Characteristics}

\section{PLACEMAKER SOFTWARE}

The software supports the PlaceMaker method of analysis and all its phases [37]. The PlaceMaker method was conceived in 2001 and has been continuously elaborated and enriched during the 


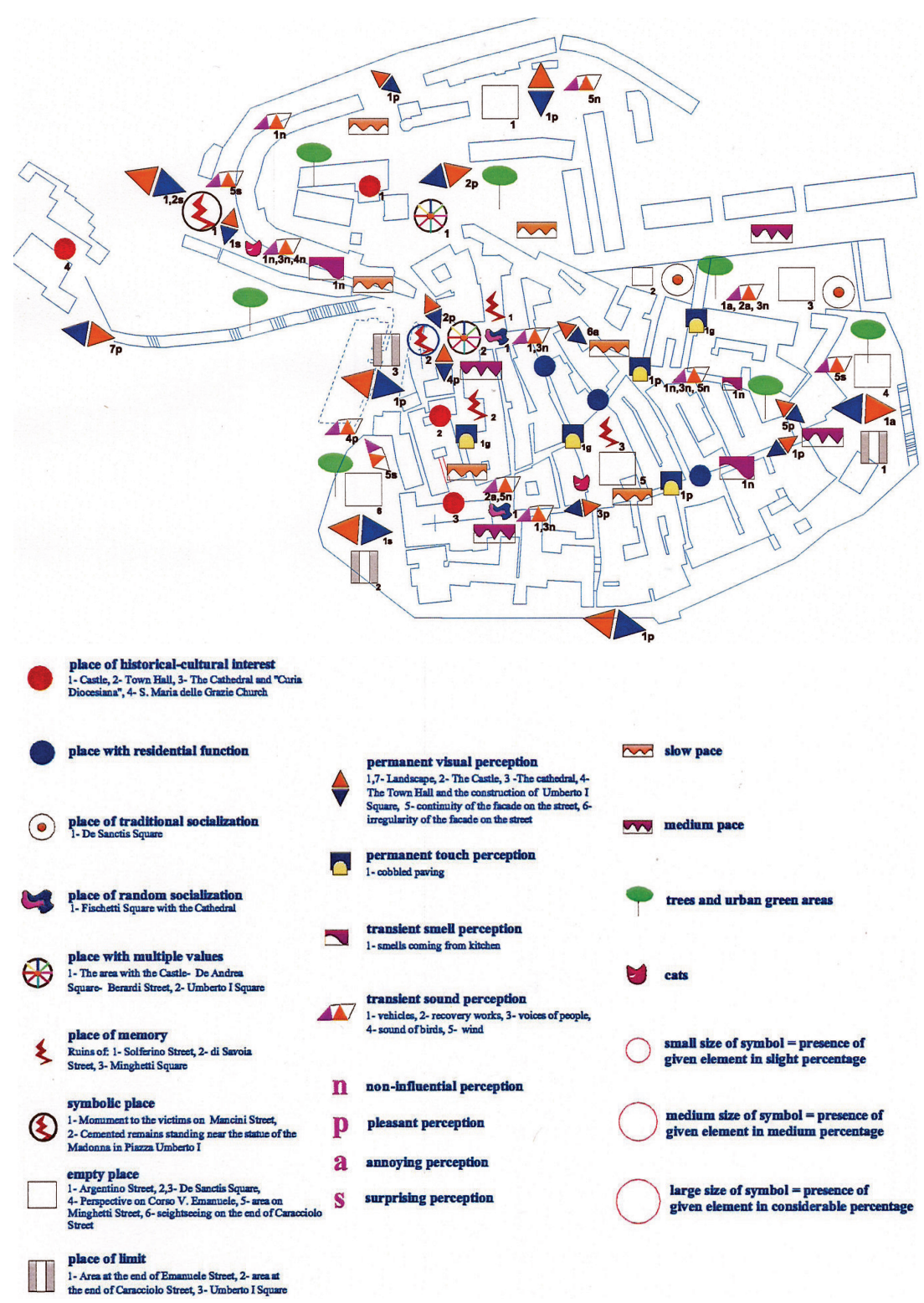

Figure 19: Marichela Sepe, complex map with PlaceMaker method.

experimentation started in 2002. The case studies have investigated urban areas in Europe and beyond which are highly representative of the cities in which they are located, revealing differences and points in common. In the latter cases, on one hand, it was possible to test the various tools used in the different phases of the relief and, on the other, we verified the efficiency of the timing and the products of the various phases, while identifying the need for some integrations. This experimentation has shown the necessity of a software tool able to support the collection and management of the data, the implementation of the phases of PlaceMaker method and the construction of the interactive complex maps. 
Furthermore, the proposed software, currently under development, connects and communicates the multimedia information contained in the complex maps and gives value and significance to those data. The prime users of the software tools are: urban planners, administrators, citizens and tourists. With PlaceMaker software, it is possible to represent and read the places of a territory through the realization of interactive dynamics and multimedia maps. The representation of the places is realized by means of the insertion in maps of symbols connected to multimedia schedules that can be continuously updated. Regarding the three criteria referred to in Section 1 - scientificity, updating and timing - the main characteristics of the software are flexibility, facility and rapidity of use, strong graphical impact, and indexing of the results. The flexibility makes it possible to conserve, manage, modify and update the multimedia data that are necessary for the creation of the multimedia schedule, connected to the symbols placed on the maps. With simple and fast operations, the creation of the maps takes place on the basis of an official traditional cartography or other kinds of maps. In map construction, cartography can be made easier by tracing the contours through the use of lines, tracing filled parts through the use of areas, or leaving these complete. The map is therefore constituted by a cartographic base on which are inserted a series of symbols to which the multimedia database is associated. The multimedia database connected to the symbols contains the data collected in the different phases of the PlaceMaker method and, in particular, written texts, schedules, images, planimetries, maps and audiovisuals. Once the symbols are inserted on the map, these can be modified, moved or eliminated. In fact, PlaceMaker software possesses a database that allows the creation and modification of the categories of symbols of the PlaceMaker method, used for the entire map. Every database series of categories contains one to which the symbols belong: the areas and the lines are defined according to category, name, colour and thickness; the symbols are defined according to category, name and dimension. The software contains a basic number of categories of symbols related to the PlaceMaker method, but it is possible to create others. Once the database of categories has been constructed, the symbols can be positioned on the base map, making it possible to connect the information referred to the places with the related multimedia schedule. It is also possible to connect the symbols with other symbols, maps or internet addresses. The partial maps and the created symbols for the final complex map can be overlapped and connected, the final product being characterized by a strong graphical impact. In order to facilitate the construction of the final complex map and consultation of the information when many data are present on one map, it is possible to decide the categories of areas, lines and symbols that must appear on the map and overlap the different maps in transparency. In order to render the results of the analysis objective and useful to sustainable urban construction, PlaceMaker software connects the symbols of the complex map to numerical indices that allow the calculation of the sustainability of the identity, the quality, the potentiality and the weakness of the places represented in the map.

\subsection{The construction of the system of symbols}

The PlaceMaker method envisages the construction of a system of symbols for each partial map and for the final complex map. In the interests of brevity, we shall now discuss the system of symbols created for the complex map. The form of the symbols is strictly related to the meaning of the different kinds of places (see Fig. 20). It has been created bearing in mind two components: the signs developed during the graphical relief related to the second phase of the PlaceMaker method and the comparison of the sign with the meaning [38]. Another criterion adopted concerns the size of the symbols; the three sizes (small, medium and large) refer to the various percentages quantifying the presence of that given element (low, medium or high). We also matched the symbols with a number in order to indicate in the legend the element to which it referred. Furthermore, it was decided to summarize the feelings 
about the elements of perception in terms of 'non-influential, pleasant, annoying', and place beside each number the first letter of these words. The strong and contrasting colours that were used aim to attract and activate the visual perception and overall perceptions. Regarding the basic system of symbols for the final complex map, the full closed circles are symbols which were chosen in order to visualize the concept of place, linked to the idea of a circumscribed area. These symbols present places of historic and cultural interest, with a residential function, commercial function, mixed functions (e.g. residential/commercial, touristic/commercial); places of traditional socialization. For the places of casual socialization, we chose an imprecise curved shape, which recalls the idea of aggregation. The uncoloured square is used to recall the sense of empty space. The concave-convex shape of space suggests a place that receives and rejects at the same time. The square with the coloured vertical bands and in the centre an uncoloured band is used to indicate a strong limit and/or boundary. For the symbols connected to perception, two fundamental types were created: one for permanent perceptions and one for transient ones, the former being denoted by symbols with a full line and colour, the latter with a dotted line and half-coloured. The 10 perception symbols recall shapes of body parts are connected to the respective actions of seeing, touching, smelling, feeling and tasting. For the symbols denoting pace, we used signs that recall the speed to which they refer and the perceptions that derive from them. For the symbols of the natural elements and animals, we used the stylized real reference image. Each map is integrated with symbols referring to the specific places and elements evoked. When reading the map, one must pay attention to the size of the symbols (indicating the elements' quantitative presence), their meaning as given in the legend, and the meaning that may derive from the proximity of two or more symbols (see Fig. 19). For example, a large symbol indicating a place of traditional socialization (such as a square) shows that the square itself is large. But if this symbol is close to the one indicating an empty place, this means that although the place should serve for socialization, in reality it does not function as such. As already stated, in order to show all the data that have led to the choice of that particular symbol, and so as not to disperse the complexity of the information, each symbol is linked by the PlaceMaker software to a multimedia schedule.

\begin{tabular}{|c|c|c|c|c|c|c|c|c|c|c|c|}
\hline Low & Medium & High & & Low & Medium & High & & Low & Medium & High & \\
\hline - & - & 0 & $\begin{array}{l}\text { place of } \\
\text { historical-cultural } \\
\text { interest }\end{array}$ & $\Delta$ & $\nabla$ & $\nabla$ & $\begin{array}{l}\text { permanent visual } \\
\text { perception }\end{array}$ & & & & \\
\hline - & - & 0 & $\begin{array}{l}\text { place with commercial } \\
\text { function }\end{array}$ & 4 & $\theta$ & $\hat{\nabla}$ & $\begin{array}{l}\text { trassient tisulal } \\
\text { perceptiton }\end{array}$ & $\infty$ & 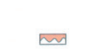 & 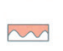 & slow pace \\
\hline$\bullet$ & - & 0 & $\begin{array}{l}\text { 施lace with residential } \\
\text { function }\end{array}$ & & ก & ( & & $m$ & $m$ & $\omega$ & regular pace \\
\hline - & - & P & $\begin{array}{l}\text { place with offices and } \\
\text { residences }\end{array}$ & 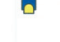 & a & 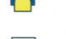 & perception & & & & \\
\hline$\odot$ & $\odot$ & - & $\begin{array}{l}\text { place of traditional } \\
\text { socialization }\end{array}$ & 早 & ๑ & له & $\begin{array}{l}\text { tratasint touch } \\
\text { perception }\end{array}$ & $\infty$ & $\infty$ & m & nectic pace \\
\hline - & (2) & (8) & $\begin{array}{l}\text { place of new } \\
\text { socialization }\end{array}$ & = & च & a & $\begin{array}{l}\text { peremanent smell } \\
\text { perception }\end{array}$ & $\varphi$ & 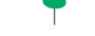 & T & 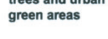 \\
\hline$\approx$ & $\approx$ & 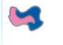 & $\begin{array}{l}\text { place of random } \\
\text { sociallzzation }\end{array}$ & ब & a & a & $\begin{array}{l}\text { transiont smoll } \\
\text { percoption }\end{array}$ & $d$ & $d$ & d & pigeons \\
\hline$\square$ & $\square$ & $\square$ & empey place & - & - & 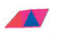 & $\begin{array}{l}\text { permanent sound } \\
\text { perception }\end{array}$ & & $\mathrm{n}$ & & 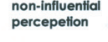 \\
\hline च & U & | & $\begin{array}{l}\text { place that receives and } \\
\text { reject }\end{array}$ & $\Delta$ & $\Delta$ & $\Delta$ & $\begin{array}{l}\text { transient sound } \\
\text { perception }\end{array}$ & & p & & $\begin{array}{l}\text { pleasont } \\
\text { perception }\end{array}$ \\
\hline 미 & [1] & 四 & place of Ilimit & $v$ & $v$ & $\checkmark$ & $\begin{array}{l}\text { perranannt tasto } \\
\text { perception }\end{array}$ & & a & & $\begin{array}{l}\text { perception } \\
\text { pers }\end{array}$ \\
\hline$\therefore$ & 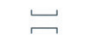 & 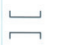 & $\begin{array}{l}\text { place open towards the } \\
\text { outside }\end{array}$ & w & उ & $B$ & $\begin{array}{l}\text { transient taste } \\
\text { perception }\end{array}$ & & & & \\
\hline
\end{tabular}

Figure 20: The system of symbols. 


\subsection{Mapping with PlaceMaker software}

The characteristics of PlaceMaker software make it possible to improve the use of the method and add some important functions useful to support the analysis and design of sustainable urban landscapes. In the following, the modalities with which the sofware supports PlaceMaker method are illustrated. The PlaceMaker method is constituted by five phases and a Phase 0 that consists in the construction of the grid required for the operations which are to be implemented later. With the use of PlaceMaker software, it is possible to collect different kinds of database to contain the different types of data collected: data from the anticipatory analysis (sketches, poems, collages, etc...); denominative and perceptive (through words), graphical (signs and symbols), photographic (fixed images), video (moving images) reliefs; the elements deduced from the study of traditional planimetries (graphic signs, symbols, etc...); the questionnaire given to visitors to the places in question (sketches, words, etc...). Once a decision has been made regarding which categories of elements to analyse and the corresponding measurement parameters, within one year taken as the reference period for urban changes, the days which are the most significant and the time slices for the relief need to be connected to the symbols. The PlaceMaker software allows the construction of the map following the different categories and measurement parameters selected. Every phase produces a map with the multimedia symbols and the related schedule. Each of the first four phases produces a partial map with symbols and the related schedule; the fifth phase produces the final complex map with symbols and related schedule of syntheses.

The first phase is devoted to an anticipatory analysis aimed at a primary investigation of the place; after the preliminary choice of the city and of the part or parts to be analysed, the ideas about that particular area can be described with the help of any type of instrument or tool of expression, using the information known before the first inspection. These notes can be represented in different ways and the result of this phase will be a map of the emerging ideas. In this phase, the data of the expectations can be inserted on the map using written text, drawings (sketch or other), images and video, always referred to a specific area. With the PlaceMaker software it is also possible to insert the text directly in word format or to make a sketch in electronic format. Once the analysis of the expectations has been carried out with a synthesis operation, it is necessary to assimilate a text or an image or other to a symbol; if the database does not contain the adequate symbol an appropriate one must be created. Such data are necessary in order to construct the multimedia schedule to connect to the symbols. Once the symbols have been inserted on the map the first phase is concluded.

The second phase is that of the five reliefs (surveys). The software makes it possible to collect the different data of those reliefs. The first relief, the denominative one, consists in the collection of the data regarding constructed elements (presence of monuments, buildings, etc.), natural elements (presence of urban green areas, trees, animals, etc.), transportation mode (presence or passing of cars, buses etc...), people (presence of tourists, residents, etc.). The localization of all these elements and the kind and amount, expressed as a low, medium, or high percentage, are indicated. In addition to the denominative data base there is a cognitive one which constitutes a kind of flexible input, where it is possible to insert elements which are not decided previously, but deduced during inspection. The second relief is perceptive; a survey is carried out of the smell, sound, taste, touch, and visual sensations, and of the global perception, focusing on the localization, type, amount (present in low, medium, high percentage) and the quality (non-influential, pleasant, annoying perceived feeling). The survey of the amount and quality of the data, the three options regarding, respectively, the percentage of presence and the feelings induced, are intended to summarize the processing of data that can however be extended during collection. The next relief is the graphical one that consists in sketching the places; the sketches will represent the area in question according to a visual-perceptive standpoint 
and will be supported by annotations where necessary. This operation constitutes a preliminary study for the construction of the graphical symbols for the complex map. Photographic and video reliefs of the whole study area are carried out, taking care to record the state of the facts rather than an interpretation of the places. The product of the five reliefs is a map visualizing the results obtained from the different reliefs. Once the five reliefs have been carried out, in addition to the nominal and cognitive database, also the sketches in digital format, the images and the video are inserted in PlaceMaker software. A second partial map with symbols and related multimedia schedule is constructed from the information deduced from the second phase.

The third phase involves the analysis of the traditional cartography of the selected sites of the city. The types of maps used in this phase, and which the software has to acquire, derive from different disciplines and depend on the nature of the place; the study is effected at the urban scale, in order to identify the characteristic elements and their relationships with that particular area, and at the aerial scale, in order to identify the relationships between the site and the whole city. The result of this phase is a map with the identification of the components required for the description of the site that can be found only through a traditional planimetrical reading. The symbols of this map will be associated using PlaceMaker software with a schedule containing the symbols of the two traditional maps.

The fourth phase is that of the questionnaire given to visitors to the area with the aim of gaining an idea of the place perceived by those who are not involved in the study and are not specialists in related fields, but only perceive the site as users, at various levels: the inhabitant, the passer-by, the tourist. The questionnaire consists of questions asked on the basis of images of the area and an inspection visit with the interviewee. The information deduced from the questionnaire will be transferred on the fourth partial map that, like the previous ones, will constitute the basis for the construction of the complex map. The schedule associated to the symbols with PlaceMaker software will be constituted mostly from images and written texts.

The fifth phase involves assembling the collected information. In this phase, we test the maps produced and the congruence of the collected data, and choose the useful elements to construct the final complex map. The recorded data represent the basis for the construction of the graphical system of symbols and the related multimedia schedule. Then, by suitably overlapping the maps obtained from the previous phases, with PlaceMaker software it is possible to produce the final complex map. The symbols created for the construction of the complex map illustrated above, as well as being connected to the database, are translated by the software into numerical indices to allow the calculation of data useful for the study of the sustainability of the places, such as liveability, wellbeing and chaoticity [39]. Finally, the map can be elaborated in relation to the changes that happen on the territory through the updating of the multimedia database and symbols. Figures 21 and 22 show two windows of the software regarding a complex map and a multimedia schedule related to the symbolic place.

\section{CONCLUSIONS}

The new structure of the contemporary city is unpredictable and complex, especially given the continuous mix of cultures which brings new elements to the already multiple urban landscapes, breaking, mixing and recomposing the complexity of urban life. This study, carried out in the framework of a convention between Consiglio Nazionale delle Ricerche and Dipartimento di Progettazione Urbana e di Urbanistica, Università di Napoli Federico II, has investigated new methods for analysing the urban landscape and new tools to represent it in order to identify the elements of the present-day urban identity, which are able to cause cultural changes and support the sustainable city construction. The questions connected to the analyses which study aspects not univocally translatable into objective facts regard in particular three criteria: scientificity, updating capability and timing. It is also observed 


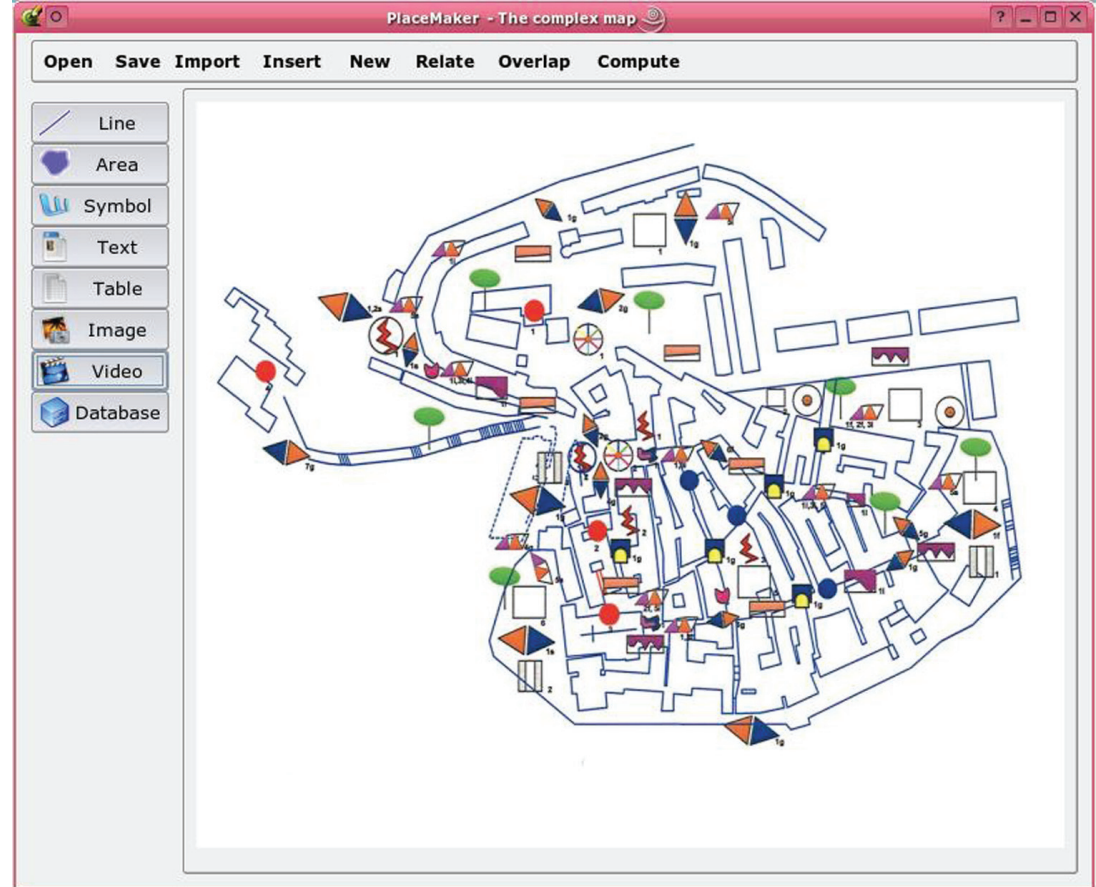

Figure 21: PlaceMaker software, window with the complex map.

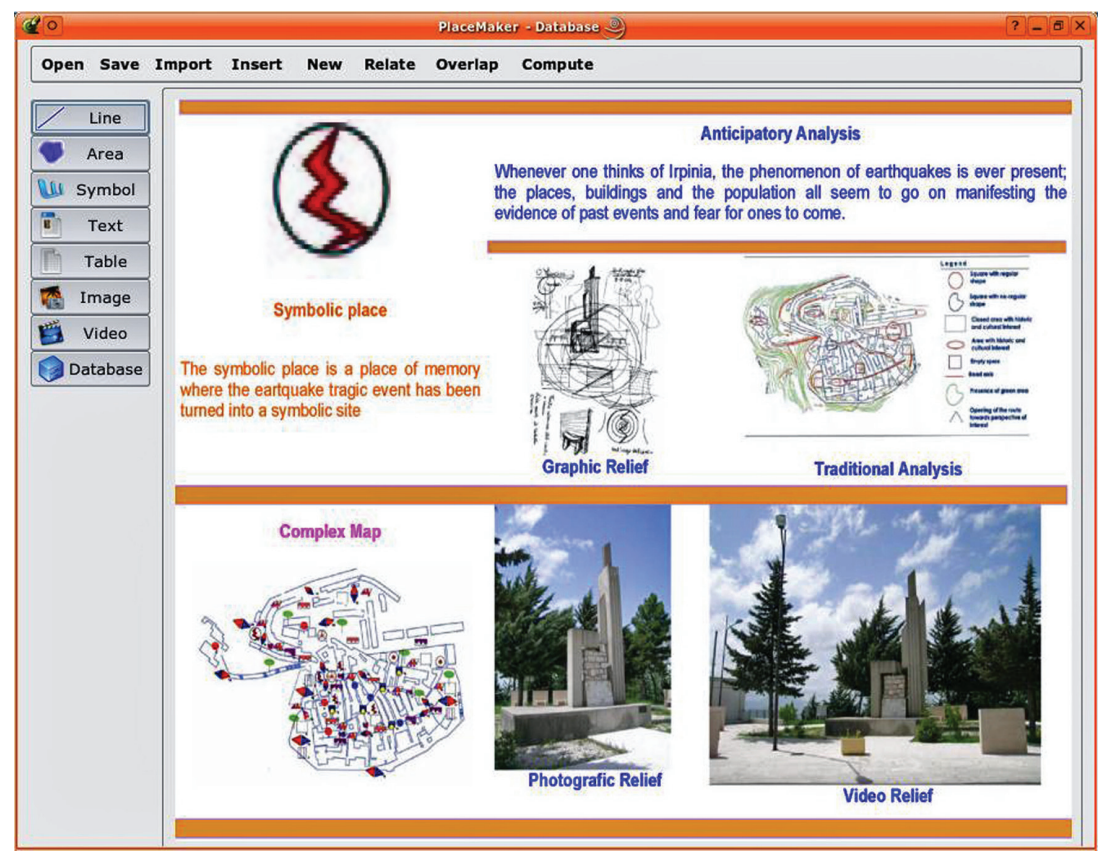

Figure 22: PlaceMaker software, window with the multimedia schedule. 
that a category comprising virtual, lateral and nomadic approaches uses atlases, maps and video in order to represent the products of specific analyses, while another category, comprising the multiscale and configurational approaches, uses software tools to support the collection and management of the data. The study of these approaches has motivated the development of a new approach, that can be defined complex-sensitive, which features in the first category as regards the used methodological approach and in the second as regards the support tool to the analysis. The main method of analysis that represents this kind of approach is represented by the PlaceMaker method. This is a methodology for analysing the urban landscape designed to identify elements that do not feature in traditional mapping and which constitute the contemporary identity of the places, representing them in a map that renders those places intelligible. Furthermore, in order to support PlaceMaker method and all its phases, a software tool, currently under elaboration, was proposed. With the proposed PlaceMaker software, it is possible to represent and read the places of a territory through the realization of interactive dynamics and multimedia maps. The main characteristics of the software are: flexibility, facility, and rapidity of use, strong graphical impact, and indexing of the results. The symbols created for the construction of the complex map, as well as being connected to the database are translated into numerical indices to allow the calculation of data useful for the study and project of the sustainability of the urban landscapes, such as liveability, well-being and chaoticity.

The difficulties that have been encountered in developing the PlaceMaker software concern three aspects which are closely interconnected: planning, realization and modes of use, and users typology. The overall work on planning PlaceMaker software is proceeding towards a satisfactory outcome. Specific problems concern, on one hand, the intention to make the tool as simple as possible without banalizing the results of the analyses; on the other, the intention to make the multimedia schedules clear and attractive in terms of the graphics. In the realization, thought is being given to whether it is preferable to create software able simply to manage multimedia data which have been defined using other software or software which comprises the facilities for fulfilling all the requisites of creation, assimilation and modification, as well as managing the multimedia data. The solution to this problem involves decisions touching on costs and modes of use, as well as the typology of users. Creating software which is able simply to manage data has a lower cost and is the preferred option for software houses, enabling them to work on modifying a product which already exists. It is not, however, the best solution in terms of mode of use and the time involved, since the user would be obliged to master a range of software packages (CAD, image management, video, etc.) in addition to the PlaceMaker software, rather than simply the latter. What is more, even with modifications, the software packages currently in the market would not provide the necessary requisites. Software able to contain and manage all the environments may well prove easier to use and more useful because it makes it possible to store all the data in the original format and use them in a dynamic and flexibile fashion. On the other hand, it will require a long time to elaborate and be very costly. Turning to the typologies of users, in principle the prime users for the PlaceMaker method and software should be urban planners, administrators, citizens, tourists and anyone involved in sustainable city construction. This latter aim involves many different figures at different levels. The development of the software has to take into account these differences, leaving room for various possible means of use. For those who carry out the analyses and draw up the maps, the software must make it possible to perform all the operations which support the elaboration of the results of the PlaceMaker method and their dynamic use in the complex planning of urban landscapes by technical experts and administrators. Nonetheless, it is obvious that the software is only meant to back up the PlaceMaker method, not to replace it, and the operations and products envisaged in the various phases of the method are effected by the field work and experimentation carried out by designated professional figures. For all those who are not interested in carrying out analyses or drawing up maps, the PlaceMaker software must 
make it possible to read and navigate the complex maps in an active manner. The goal is to get to know places in the urban landscape, and also to be able to add new elements, creating a tool that fosters participation on the part of the citizen and an in-depth knowledge on the part of the tourist.

\section{ACKNOWLEDGEMENTS}

The author thanks the reviewers for their useful suggestions.

\section{REFERENCES}

[1] Whitehand, J.W.R. \& Larkham, P.J., Urban Landscape, Routledge: London and New York, 1992.

[2] Hauptmann, D., ed., Cities in Transition, Rotterdam Publishers: Rotterdam, 2001.

[3] Orum, A.M. \& Chen X., City and Places, Blackwell: Oxford, 2003.

[4] Borden, L., Herr, J. \& Rendell, J., eds, The Unknown City, MIT Press: Cambridge, MA, 2002.

[5] Banerjee, T. \& Southworth, M., eds, City Sense and City Design, MIT Press: Cambridge, MA, 1990.

[6] Groupe E2, Exploring the Urban Condition, ACTAR: Barcellona, 2002.

[7] Gausa, M. et al., The Metapolis Dictionary of Advanced Architecture, ACTAR: Barcelona, 2003.

[8] Cassatella, C., Iperpaesaggi, Testo e Immagine: Torino, 2001.

[9] Sepe, M., Complex mapping of the urban landscape: new methods and complex maps. Proc. of IPHS International Planning History Conference, Barcelona, 2004.

[10] Mitchell, W.J., City of Bits, MIT Press: Cambridge, MA, 1996.

[11] Boeri, S., USE, Skira: Milano, 2003.

[12] Careri, F., New Babylon, Una Città Nomade, Testo e Immagine: Torino, 2001.

[13] MVRDV, The Regionmaker. Rheinruhrcity, Hatje Cantz: Ruit, 2002.

[14] Hillier, B. \& Stutz, C., New methods in space syntax. Urban Design, 93, pp. 32-33, 2005.

[15] Ratti, C., Sevtsuk, A., Huang, S. \& Pailer, R., Mobile landscapes: Graz in real time. Proc. of the 3rd Symposium on LBS \& TeleCartography, Vienna, Austria, 2005.

[16] Gregory, P., Territori della complessità, Testo e Immagine: Torino, 2003.

[17] Lynch, K., The Image of the City, MIT Press: Cambridge, MA, 1960.

[18] Cullen, G., Il paesaggio urbano, Edizioni Calderoni: Bologna, 1976.

[19] Garcìa Pérez, J.D., Ascertaining landscape perceptions and preferences with pair-wise photographs: planning rural tourism in Extremadura, Spain. Landscape Research, 27(3), pp. 297-308, 2002.

[20] Vale, L.J. \& Warner Jr, S.B., eds, Imaging the City, Center for Urban Policy Research: New Jersey, 2001.

[21] Krause, C.L., Our visual landscape. Managing the landscape under special consideration of visual aspects. Landscape and Urban Planning, 57, pp. 239-254, 2001.

[22] Stalker, Attraverso i territori attuali/À travers les territoires actuels, Jean Michel Place: Paris, 2000.

[23] Andreotti, L. \& Xavier, C., Theory of the Dèrive and Other Situationist Writings on the City, MACBA/ACTAR: Barcelona, 1996.

[24] IAAC Metápolis, Hipercatalunya, Research Territories, Actar: Barcelona, 2003.

[25] Hillier, B., The hidden geometry of deformed grids: or, why space syntax works, when it looks as though it shouldn't. Environment and Planning B - Planning \& Design, 26(2), pp. 169-191, 1999. 
[26] Ratti, C., Suggestions for developments in space syntax. Space Syntax, ed. A. Van Nes, Delft University Press: Delft, 2005.

[27] Lassus, B., Jardins Imaginaires, Les Presses de la Connaissance: Paris, 1977.

[28] Denis, C. \& Daniels, S., The Iconography of Landscape: Essay on the Symbolic Representation, Design and Use of Past Environments, Cambridge University Press: Cambridge, MA, 1988.

[29] Gospodini, A., Urban space morphology and place-identity in European cities; built heritage and innovative design.Journal of Urban Design, 9(2), pp. 225-248, 2004.

[30] Miller, N., Mapping the City, Continuum: New York, 2003.

[31] Forrester, J.W., Urban Dynamics, MIT Press: Cambridge, MA, 1969.

[32] Gandelsonas, M., The Urban Text, MIT Press: Cambridge, MA, 1991.

[33] Sepe, M., Complex analysis for the sustainable planning and construction of the place identity: the Sensitive Relief method. International Journal of Sustainable Development and Planning, 1(1), pp. 14-31, 2006.

[34] Ayeni, O.O., Saka, D.N. \& Ikwuemesi, G., Developing a multimedia gis database for tourism industry in Nigeria. Proc. of ISPRS, Istanbul, 2004.

[35] Graziano, L., Il sistema autore per creare progetti interattivi on-line. Proc. of INPUT '99, Venezia, 1999.

[36] Marinelli, A.M., Uno strumento interattivo per il progetto urbano. Proc. of INPUT'99, Venezia, 1999.

[37] Sepe, M., The PlaceMaker: a flexible and interactive tool to support the sustainable city construction and transformation. Proc. of Sustainable Planning and Development 2005, WIT Press: Southampton, pp. 1525-1534, 2005.

[38] Sepe, M., The sensitive relief method and the identity of a place. Architektura \& Urbanizmus, 39(1-2), pp. 65-80, 2005.

[39] Sepe, M., Planning with PlaceMaker: complex indices for sustainable projects. Proc. of Sustainable City 2006, WIT Press: Southampton, 2006. 